\title{
Multisector dosimetry in the immediate post-implant period: significant under dosage of the prostate base
}

\author{
Austin N. Kirschner, MD, PhD, Vythialingam Sathiaseelan, PhD, Yunkai Zhang, PhD, James David, MS, \\ John A. Kalapurakal, MD, FACR \\ Radiation Oncology, Northwestern Memorial Hospital, Chicago, USA
}

\begin{abstract}
Purpose: While there are several reports of prostate multisector dosimetry data obtained from CT or MRI scans performed at intervals ranging from 14-70 days after prostate brachytherapy (PB), there are no reports on multisector dosimetry performed in the immediate post-implant period. This study was undertaken to determine the results of prostate multisector dosimetry performed in the immediate post-implant period on day 1 post-implant dosimetry after ${ }^{125} \mathrm{I}$ PB.

Material and methods: The day 1 post-implant CT-based $V_{100}$ and $\mathrm{D}_{90}$ were determined for the prostate base (PGB) and compared to doses to the entire gland (PG), mid-gland (PMG), and apex (PA) in 75 patients who underwent ${ }^{125} \mathrm{I} \mathrm{PB}$ to a dose of $144 \mathrm{~Gy}$. Similar multisector dosimetry was also performed on the pre-implant ultrasound volume study scans of these patients.

Results: All patients had good quality implants. On day 1 post-implant multisector dosimetry there was significant under dosage of the PGB for both $\mathrm{V}_{100}$ and $\mathrm{D}_{90}$. The average magnitude of under dosage of PGB compared to PMG and PA was $17.2 \%$ and $22.7 \%$ for $\mathrm{V}_{100}$ and $44.6 \mathrm{~Gy}$ and $31.7 \mathrm{~Gy}$ for $\mathrm{D}_{90}$, respectively. On pre-implant multisector dosimetry there was no statistically significant under dosage of the PGB for $\mathrm{V}_{100}$, but the PGB $\mathrm{D}_{90}$ was significantly lower compared to PMG and PA, however, the average magnitude of under dosage was small at 12.6 Gy and 4.2 Gy, respectively.

Conclusions: This report demonstrates that similar to other reports on more delayed post-implant multisector dosimetry data, there is significant under dosage of the prostate base in the immediate post-implant period based on day 1 post-implant dosimetry. The clinical significance of this under dosage remains to be defined and further studies are warranted.
\end{abstract}

Key words: brachytherapy, CT-based dosimetry, multisector, prostate cancer.

\section{Purpose}

In patients with clinically localized prostate cancer, prostate permanent seed brachytherapy (PB) is associated with excellent biochemical control rates [1-5]. The American Brachytherapy Society consensus guidelines recommend that CT-based postoperative dosimetry is to be performed at any time between 0-60 days after PB. Many centers, including ours, perform the post-implant $\mathrm{CT}$ scan on day 0 or day 1 , as this is more convenient for the patient, allows for early identification of dosimetric problems and closes the learning loop, while the memory of the procedure is still recent. However, it is recognized that that undertaking the procedure at this time would under estimate the dosimetric parameters, because of the presence of edema $[5,6]$. The ABS has not proposed any consensus guidelines for prostate multisector dosimetry [5]. While there are several reports of prostate multisector dosimetry data obtained from CT or MRI scans performed at intervals ranging from 14-70 days after $\mathrm{PB}$, there are no reports on multisector dosimetry performed in the immediate post-implant period [7-12]. This study was undertaken to determine the results of prostate multisector dosimetry performed in the immediate post-implant period on day 1 post-implant dosimetry after ${ }^{125} \mathrm{I} \mathrm{PB}$.

\section{Material and methods}

\section{Patient selection}

The data in this study was obtained from 75 consecutive patients treated by a single radiation oncologist (JAK). These patients underwent ${ }^{125}$ I prostate brachytherapy as sole therapy to a dose of 144 Gy (AAPM TG-43) prescribed to the prostate gland planning target volume [5]. All patients had low-risk prostate cancer (stage T1c/ T2a [1992 AJCC criteria] with a Gleason score $\leq 6$, and serum PSA $\leq 10 \mathrm{ng} / \mathrm{mL}$ ). 


\section{Pre-implant volume study}

The TRUS volume study was performed jointly by the radiation oncologist and urologist using B\&K ultrasound (B\&K Medical, Herlev, Denmark) unit and a Barzell-Whitmore stepper (Barzell-Whitmore Maroon Bells, Inc., Sarasota, Fla., USA). Images were obtained at $5 \mathrm{~mm}$ intervals from base to apex of the prostate gland. Ultrasound pre-planning was performed using a urethral-sparing modified uniform loading technique, described by the Seattle Prostate Institute using the Prowess version 3.1 treatment planning system (Prowess, Chico, CA) [1,3]. The individual ${ }^{125} \mathrm{I}$ seed strength ranged from 0.293 to $0.337 \mathrm{mCi}$. The prostate clinical target volume was the outline of the prostate gland at the time of the volume study (Fig. 1). This volume was expanded by $2 \mathrm{~mm}$ anteriorly and laterally, and $5 \mathrm{~mm}$ inferiorly and no margin was applied posteriorly and superiorly to define the planning target volume (PTV).

\section{Brachytherapy implant procedure}

All patients underwent brachytherapy under spinal or general anesthesia. Prostate brachytherapy was then performed using a standard template-guided transperineal technique using preloaded needles and free seeds from the anterior to the posterior regions of the prostate gland as described earlier [13].

\section{Post-implant dosimetry}

All patients had a post-implant CT scan performed on day 1 after brachytherapy. The CT scans were performed at $3 \mathrm{~mm}$ intervals, using $3 \mathrm{~mm}$ slice thickness and post-implant dosimetry was performed using the Prowess version 3.1 treatment planning system (Prowess, Chico, CA, USA). The prostate gland was outlined by a single radiation oncologist (JAK). A good quality prostate implant was defined by $\mathrm{V}_{100} \geq 80 \%$ on day 1 post-implant dosimetry $[3,5,12]$.

\section{Prostate multisector dosimetry}

One of the major impediments to the uniform conduct of prostate multisector dosimetry is the lack of consensus in the definition of the various prostate sectors. A number of reports have described brachytherapy doses to the prostate base, mid gland, and apex using varying definitions [7-12]. In one report, the base was represented by the two superior most CT slices and the apex was designated as the two inferior most CT slices [7]. In other reports the prostate gland was divided supero-inferiorly into two or three equal quadrants [8-10]. In two recent reports, the prostate was divided into three equal quadrants (base, mid gland, apex) along its length, and then each quadrant was divided into four sectors by using perpendicular or oblique dividers for a total of 12 sectors [11,12]. In
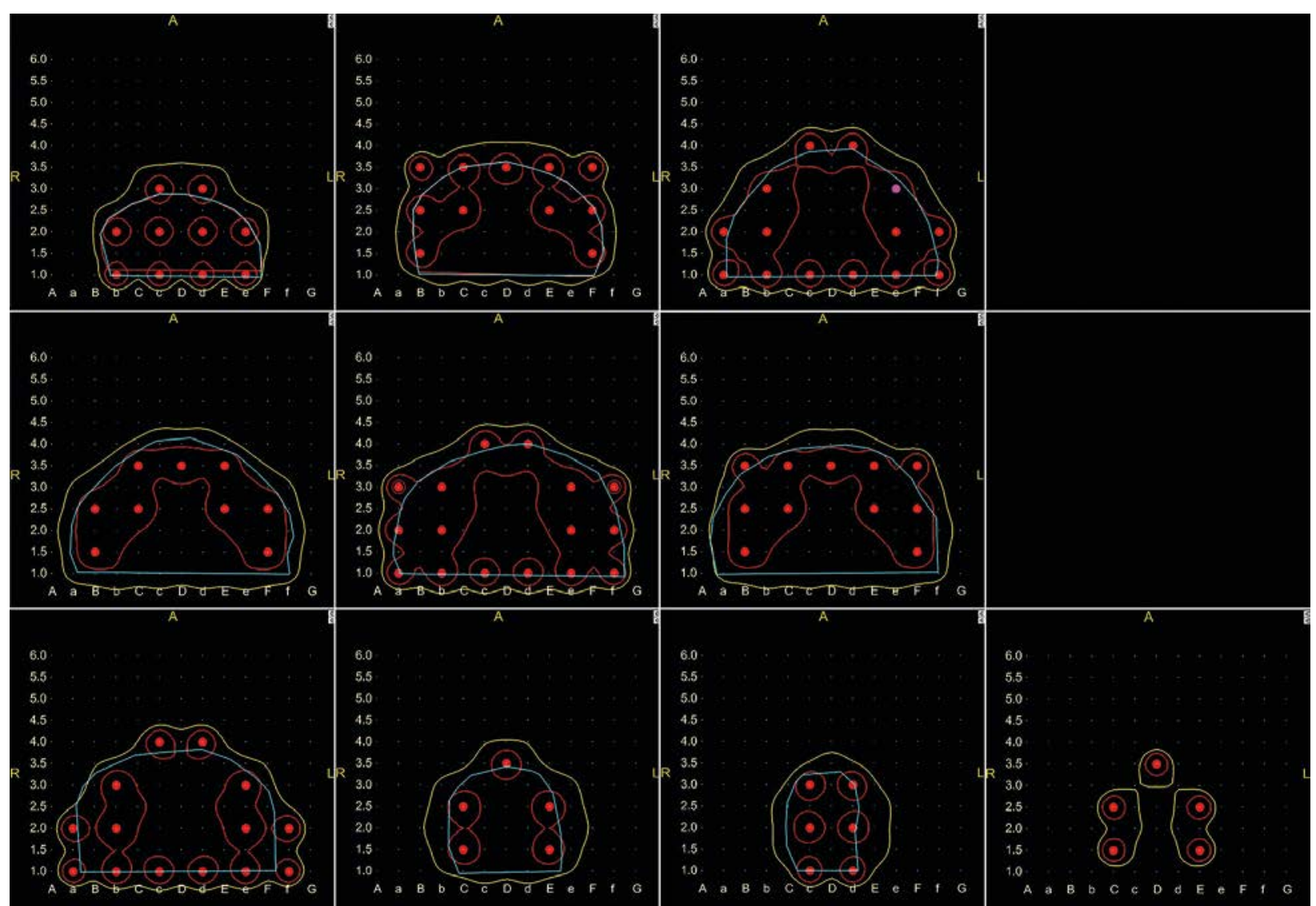

Fig. 1. Pre-implant ultrasound dosimetry axial scans spaced $5 \mathrm{~mm}$ apart. The prostate gland is outlined in cyan, $144 \mathrm{~Gy}(100 \%)$ isodose line in yellow and $216 \mathrm{~Gy}(150 \%)$ isodose line in red. The multisector dosimetric parameters are: Base (top row): $\mathrm{D}_{90}=$ $=168.3 \mathrm{~Gy}, \mathrm{~V}_{100}=99.8 \%$; Mid gland (middle row): $\mathrm{D}_{90}=177.1 \mathrm{~Gy}, \mathrm{~V}_{100}=100.0 \%$; Apex (bottom row): $\mathrm{D}_{90}=162.2 \mathrm{~Gy}, \mathrm{~V}_{100}=98.9 \%$ 
another report by $\mathrm{D}^{\prime}$ Amico et al. that reviewed 104 radical prostatectomy specimens for anterior basal cancer involvement to define implant volume in low-risk prostate cancer, the superior-most $1 \mathrm{~cm}$ of the gland was defined as the prostate base [14]. Given the lack of consensus, we have utilized this superior-most $1 \mathrm{~cm}$ definition for defining the prostate base on pre-implant ultrasound (US) and post-implant CT scans. While the D' Amico report defined the prostate apex as the distal $5 \mathrm{~mm}$ of the gland, we used the distal $1 \mathrm{~cm}$ to define the apex similar to the base definition, because of the volumetric and dosimetric limitations imposed by using just 1 or 2 axial images on the ultrasound or CT scans. The mid gland was defined as the region in between the base and apex on pre- and post-implant ultrasound and CT images. As can be observed in Figures 1 and 2, the definition of the prostate base, mid gland, and apex used in this report is quite similar to the definition used by Merrick et al., in which the prostate was divided into three equal segments longitudinally [12]. The Pre-implant $\mathrm{V}_{100}$ and $\mathrm{D}_{90}$, and Post-implant $\mathrm{V}_{100}$ and $\mathrm{D}_{90}$, and $V_{150}$ were determined for the entire gland, prostate base, mid gland, and apex in 75 consecutive patients [5]

\section{Statistics}

The Pre-implant $\mathrm{V}_{100}$ and $\mathrm{D}_{90}$, and Post-implant $\mathrm{V}_{100}$ and $\mathrm{D}_{90}$, and $\mathrm{V}_{150}$ were determined for the entire gland, prostate base, mid gland, and apex. These dose values for the entire gland and prostate sub-regions were compared using a two-tailed Student's $t$-test to determine potential areas of under dosage on pre-implant or post-implant dosimetry. A $p$ value of $<0.05$ indicated statistical significance.

\section{Results}

\section{Pre-implant dosimetry}

The mean $V_{100}( \pm S D)$ for the entire prostate gland (PG), prostate base (PGB), prostate mid-gland (PMG), and prostate apex $(\mathrm{PA})$ was $99.2 \%( \pm 1 \%), 98.9 \%( \pm 1 \%)$, $99.5 \%( \pm 1 \%)$, and $98.5 \%( \pm 4 \%)$, respectively. The mean $\mathrm{D}_{90}( \pm \mathrm{SD})$ for the PG, PGB, PMG, and PA was $174.1 \mathrm{~Gy}$ $( \pm 8.14), 166.1 \mathrm{~Gy}( \pm 6.63), 178.7 \mathrm{~Gy}( \pm 8.25)$, and 170.3 $( \pm 1.42)$, respectively. There was no statistically significant under dosage of the prostate base with respect to $\mathrm{V}_{100}$ compared to the entire gland ( $p$ value 0.20 ) and apex ( $p$ value 0.36 ). The magnitude of under dosage of the base $\mathrm{V}_{100}$ compared to mid gland was $0.6 \%$ ( $p$ value $<0.01$. The base $D_{90}$ was statistically significantly lower compared to the mid gland and apex, however, the average magnitude of under dosage was small at 12.6 Gy and 4.2 Gy, respectively (Table 1). Multisector dosimetry for $\mathrm{V}_{100}$ and $\mathrm{D}_{90}$ of the prostate mid gland and apex revealed no significant under dosage in these regions.

\section{Post-implant dosimetry}

The mean volume $( \pm \mathrm{SD})$ of the PG, PGB, PMG, and PA was $50.9 \mathrm{cc}( \pm 14.0), 14 \mathrm{cc}( \pm 3.8), 30.2 \mathrm{cc}( \pm 11.2)$, and $6.8 \mathrm{cc}( \pm 2.3)$, respectively. All patients had good quality implants based on $V_{100} \geq 80 \%$ to the entire prostate gland per the Seattle Prostate Institute guidelines. The mean $\mathrm{V}_{100}( \pm \mathrm{SD})$ for the PG, PGB, PMG, and PA was $90.2 \%( \pm 6)$, $77.9 \%$ ( \pm 14$), 95.1 \%( \pm 5)$, and $90.6 \%$ ( \pm 12 ), respectively. The mean $\mathrm{D}_{90}$ for PG, PGB, PMG, and PA was 148.7 Gy $( \pm 22.1), 125.7 \mathrm{~Gy}( \pm 23.3), 170.3 \mathrm{~Gy}( \pm 26)$, and $157.4 \mathrm{~Gy}$ $( \pm 29.5)$, respectively. The mean $\mathrm{V}_{150}$ for the PG, PGB, PMG, and PA was $52.8 \%( \pm 15.5), 34.8 \%( \pm 16.6), 61.1 \%( \pm 17.7)$, and $53.5 \%( \pm 22.0)$, respectively. There was significant under dosage of the prostate base relative to the entire prostate gland, mid-gland, and apex for mean $\mathrm{V}_{100}(p$ value $<0.01)$ and $\mathrm{D}_{90}(p$ value $<0.01)$. The average magnitude of under dosage for the prostate base compared to the midgland and apex was $17.2 \%$ and $22.7 \%$ for $\mathrm{V}_{100}$, and $44.6 \mathrm{~Gy}$ and $31.7 \mathrm{~Gy}$ for $\mathrm{D}_{90}$, respectively (Table 2). Multisector dosimetry for $\mathrm{V}_{100}$ and $\mathrm{D}_{90}$ of the prostate mid gland and apex revealed no under dosage in these regions. To the contrary, there actually was a significant increase in $V_{150}$ in these regions by $26.3 \%$ and $18.7 \%$, respectively, compared to the prostate base $(p$ value $<0.01)$.

\section{Discussion}

There is considerable variation in the practice of preand post-implant dosimetry [5,15-17]. The pre-implant dose coverage of the prostate gland $\left(\mathrm{V}_{100}\right)$ is generally $>95 \%$. However, similar to the Vancouver series [9], our analysis of pre-implant dosimetry also demonstrated some degree of under dosage of the prostate base relative to mid gland and apex. However, in both of these reports, the magnitude of post-implant under dosage of the prostate base was significantly greater than any under dosage that was observed on pre-implant ultrasound dosimetry.

The current indicators of good quality implants such as $V_{100}$ and $D_{90}$ considers the dose coverage of $90-100 \%$ of the prostate volume by a specific dose [1-5]. While several reports have suggested a significant correlation between $\mathrm{D}_{90}$ and biochemical control $[4,17]$, others have not found such a correlation [18]. This discrepancy may in part be due to the fact that these dose-volume descriptors do not give an accurate assessment of whether the tumor bearing sectors within the prostate gland were adequately dosed or not. Although the prescribed minimum dose to the prostate gland is approximately $145 \mathrm{~Gy}$, the dose to quadrants within the prostate gland can vary from $60 \mathrm{~Gy}$ to $>500 \mathrm{~Gy}$. The clinical impact of such a variable dose distribution in the different prostate sub-regions on tumor control rates is not known [19].

To our knowledge, there have been no reports on post-implant multisector dosimetry in the immediate post-implant (day 0-1) period. At least six reports using differing definitions for the various prostate sectors has described above and have performed dosimetric analysis at intervals of 14-70 days after brachytherapy. Ash et al. performed a segmental analysis of prostate dose from CT scans on 32 patients at 6-8 weeks after ${ }^{125} \mathrm{I}$ brachytherapy. They observed that the average $D_{90}$ to the basal segments was approximately $80 \%$ compared to $90-95 \%$ to the mid-section and apex of the gland [8]. Sidhu et al. analyzed the dose to four quadrants of the prostate gland in 30 patients from CT scans performed 12-70 days after ${ }^{125} \mathrm{I}$ brachytherapy. The mean $\mathrm{V}_{100}$ for the anterior supe- 


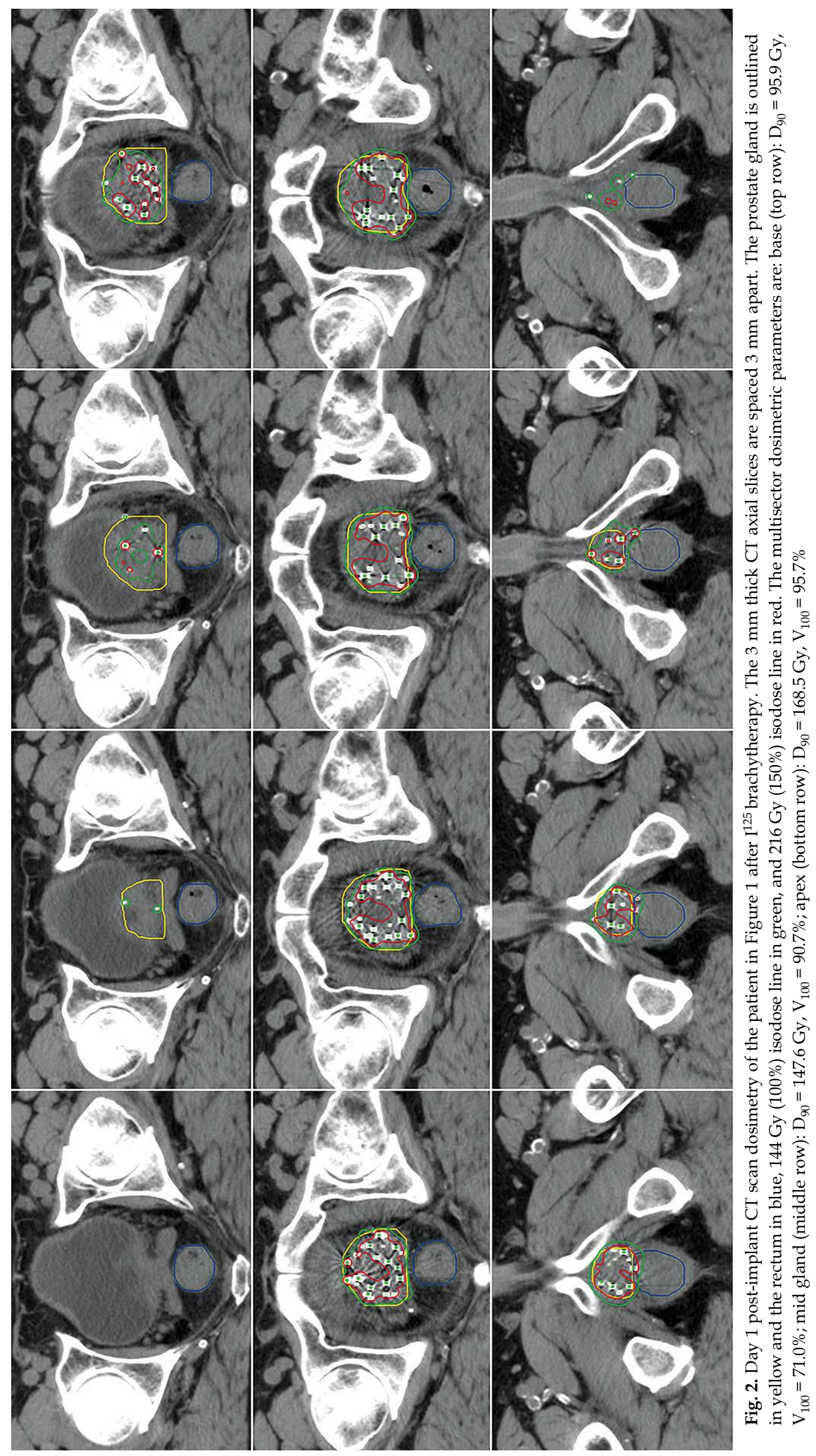


Table 1. Comparison of $\mathrm{V}_{100}$ and $\mathrm{D}_{90}$ of the prostate base relative to the entire prostate gland, mid-gland, and apex on pre-implant ultrasound multisector dosimetry

\begin{tabular}{|c|c|c|c|c|c|c|c|c|c|c|}
\hline \multicolumn{2}{|c|}{ Dosimetry parameter } & \multicolumn{3}{|c|}{ Base vs. Entire gland } & \multicolumn{3}{|c|}{ Base vs. Mid-gland } & \multicolumn{3}{|c|}{ Base vs. Apex } \\
\hline & & Base & Entire gland & $p$ value & Base & Mid-gland & $p$ value & Base & Apex & $p$ value \\
\hline \multirow[t]{2}{*}{$V_{100}$} & mean & $98.9 \%$ & $99.2 \%$ & 0.20 & $98.9 \%$ & $99.5 \%$ & $<0.01$ & $98.9 \%$ & $98.5 \%$ & 0.36 \\
\hline & SD & $1 \%$ & $1 \%$ & & $1 \%$ & $1 \%$ & & $1 \%$ & $4 \%$ & \\
\hline \multirow[t]{2}{*}{$D_{90}$} & mean & 166.1 & 174.1 & $<0.01$ & 166.1 & 178.7 & $<0.01$ & 166.1 & 170.3 & 0.02 \\
\hline & SD & 6.63 & 8.14 & & 6.63 & 8.25 & & 6.63 & 1.42 & \\
\hline
\end{tabular}

$V_{100}$ - Percentage volume of the prostate gland that receives $100 \%$ of prescription dose, $D_{90}$-dose (Gy) that treats $90 \%$ of the prostate gland volume, SD - standard deviation

Table 2. Comparison of $\mathrm{V}_{100}$ and $\mathrm{D}_{90}$ of the prostate base relative to the entire prostate gland, mid-gland, and apex on day 1 post-implant CT scan multisector dosimetry

\begin{tabular}{|c|c|c|c|c|c|c|c|c|c|c|}
\hline \multirow{2}{*}{\multicolumn{2}{|c|}{ Dosimetry parameter }} & \multicolumn{3}{|c|}{ Base vs. Entire gland } & \multicolumn{3}{|c|}{ Base vs. Mid-Gland } & \multicolumn{3}{|c|}{ Base vs. Apex } \\
\hline & & Base & Entire gland & $p$ value & Base & Mid-gland & $p$ value & Base & Apex & $p$ value \\
\hline \multirow[t]{2}{*}{$V_{100}$} & mean & $77.9 \%$ & $90.2 \%$ & $<0.01$ & $77.9 \%$ & $95.1 \%$ & $<0.01$ & $77.9 \%$ & $90.6 \%$ & $<0.01$ \\
\hline & SD & $14 \%$ & $6 \%$ & & $14 \%$ & $5 \%$ & & $14 \%$ & $12 \%$ & \\
\hline \multirow[t]{2}{*}{$\mathrm{D}_{90}$} & mean & 125.7 & 148.7 & \multirow[t]{2}{*}{$<0.01$} & 125.7 & 170.3 & \multirow[t]{2}{*}{$<0.01$} & 125.7 & 157.4 & \multirow[t]{2}{*}{$<0.01$} \\
\hline & SD & 23.3 & 22.1 & & 23.3 & 26.0 & & 23.3 & 29.5 & \\
\hline
\end{tabular}

$V_{100}$-Percentage volume of the prostate gland that receives $100 \%$ of prescription dose, $D_{90}$-dose (Gy) that treats $90 \%$ of the prostate gland volume, SD - standard deviation

rior quadrant was $78.5 \%$ compared to $90-99 \%$ for the other quadrants of the gland. Similarly, the mean $\mathrm{D}_{90}$ to the anterior superior quadrant was 130.6 Gy compared to 162-198.6 Gy in the other quadrants [9]. In another report by Maletz et al. there was significant under dosage of the anterior base $\mathrm{V}_{100}$ and $\mathrm{D}_{90}$ from MRI and CT post-implant dosimetry performed at 2-4 weeks after ${ }^{103} \mathrm{Pd}$ brachytherapy in 45 patients [10]. Similarly, Butzbach et al. reported that under dosage of the prostate base led to significantly smaller extraprostatic margin with dose coverage in the base $(2.5-3.5 \mathrm{~mm})$, compared to the mid gland and apex $(\geq 4.5 \mathrm{~mm})$ on post implant dosimetry in 22 patients 20-53 days after ${ }^{103} \mathrm{Pd}$ brachytherapy [7]. Recently Merrick et al. reported on dosimetry data on twelve prostate sectors from the Pro-Qura database performed at a median of 30 days after brachytherapy. They reported that $33 \%, 4.6 \%$, and $10.5 \%$ of all base, mid gland, and apical sectors of the prostate were considered of minimal quality with a $\mathrm{V}_{100}<80 \%$. Specifically, $59.2 \%$ of the anterior base and $30.3 \%$ of the posterior base had minimal quality dose coverage [12]. Similarly, Yoosuf et al. has shown significant under dosage of the base and anterior mid gland on CT-based 12 sector dosimetry performed 28 days after ${ }^{125} \mathrm{I}$ brachytherapy in 50 patients [11].

Our data demonstrates a similar under dosage of the prostate base in the immediate post-implant period on day 1 multisector dosimetry revealing a prostate base under dosage compared to the mid gland and apex by an average magnitude of $17.2 \%$ and $22.7 \%$ for $\mathrm{V}_{100}$, and 44.6 Gy and 31.7 Gy for $\mathrm{D}_{90}$, respectively. Our report did not find any under dosage in the prostate mid gland and apex sectors. This report adds to the existing literature on under dosage of the prostate base on post-implant dosimetry performed 2 weeks to 70 days after implantation by demonstrating that day 1 post-implant multisec- tor dosimetry also shows significant basal under dosage. An argument could be made that there would be more swelling in the immediate post-implant period, and that more errors would be associated with CT-based contouring as in this report, thus accentuating potential areas of underdosage on day 1 dosimetry. However, our results are no different from the other published reports on post-implant dosimetry using CT and MRI scans done 14-70 days after brachytherapy [7-12]. Thus, despite the lack of consistency in the definition of prostate sectors, isotopes, timing, and inter and intra-observer variability associated with post-implant dosimetry, this report and others demonstrates a broad consensus that significant under dosage of the prostate base is commonly observed on CT/MRI scans performed at intervals of 1-70 days after prostate brachytherapy [5,7-12].

There are several reasons for the under dosage of the base including loss of seeds into the bladder or periprostatic venous plexus, inferior migration of seeds during implantation, incorrect location of the needle tip relative to the base, needle drag on the prostate gland, needle splay, and errors in accurate definition of the prostate base on CT scans [10-12,20]. Another important reason could be due to the fact that unlike other quadrants of the prostate gland, a PTV margin cannot be applied superiorly to the base as seeds intended for tissues $5 \mathrm{~mm}$ superior to the base would either be lost in the urinary bladder or get implanted in the bladder wall that could result in increased urinary complications. This may also explain the slight under dosage of the base seen on pre-implant dosimetry in this report. The clinical significance of under dosage of the prostate base or anterior regions of the prostate gland is unknown. However, such under dosage has the potential to decrease tumor control rates considering that $>75 \%$ of prostate tumors are multifocal [21], 
and $5-30 \%$ of low-risk tumors may actually have higher grade and higher stages of cancer [22,23]. Further, recent reports have shown an increased incidence (20-25\%) of anterior-predominant prostate cancers in patients undergoing prostatectomy after aggressive screening [24], and involvement of all regions of the prostate gland including the base in low-risk patients undergoing template-guided saturation biopsies [25]. For all of these reasons, similar to the practice of radical prostatectomy and external beam radiotherapy, every attempt should be made to treat the entire prostate gland including the base with prostate brachytherapy.

The use of MRI scans for post-implant dosimetry may provide a more accurate definition of prostate volumes, however, this is not currently the standard of care $[5,26]$. In this report we have used a simple definition to divide the prostate into three sectors: base, mid gland, and apex that can easily be utilized for routine sector specific dosimetric analysis on pre- and post-implant US and CT scans. The clinical utility to dividing the gland into more number of sectors as done by others is uncertain, especially because of the difficulty in accurately depositing seeds, and thus delivering the dose specifically in smaller sectors of the prostate gland. In the future, prostate multisector dosimetry may have more clinical importance with the increasing use of multiparametic MRI scans for intra-prostatic tumor localization and staging [26]. The combined use of multiparametric MRI, pre and post-implant assessment of $\mathrm{V}_{100}$ and $\mathrm{D}_{90}$ for the entire prostate gland, prostate base, mid gland, and apex has the potential to improve tumor control rates by ascertaining that tumor bearing regions within the prostate gland, especially at the base are adequately treated and not under dosed after good quality brachytherapy. The measures that could be utilized to reduce under dosage intra-operatively or compensate for under dosage post-operatively, especially in patients with a high risk of tumor involvement of the base may include: the addition of a $5 \mathrm{~mm}$ planning margin superiorly into the seminal vesicles; use of unconventional seed loading to increase the number of seeds or the use of higher strength seeds at the base; use of high dose rate brachytherapy [2]; or the addition of external beam irradiation (45 Gy) to brachytherapy in intermediate and high risk patients [5]. Studies are underway at our institution to determine the prognostic significance of prostate quadrant dosimetry on biochemical outcomes.

\section{Conclusions}

This report demonstrates that there is significant under dosage of the prostate base compared to the midgland and apex in the immediate post-implant period based on day 1 post-implant dosimetry. Despite the wide variability in the practice of post-implant dosimetry with regard to timing after brachytherapy, type of scans (CT vs. MRI) and the criteria used to define prostate sectors, the areas of under dosage in the prostate gland are not randomly distributed, but are consistently observed in the basal segments of the prostate gland. Further research is warranted to investigate measures to avoid under dos- age of the prostate base and to study its potential clinical significance.

\section{Disclosure}

Authors report no conflict of interest.

\section{References}

1. Sylvester JE, Grimm PD, Wong J et al. Fifteen-year biochemical relapse-free survival, cause-specific survival, and overall survival following I-125 prostate brachytherapy in clinically localized prostate cancer: Seattle experience. Int J Radiat Oncol Biol Phys 2011; 81: 376-381.

2. Skowronek J. Low-dose-rate or high-dose-rate brachytherapy in treatment of prostate cancer - between options. J Contemp Brachytherapy 2013; 5: 33-41.

3. Sylvester J, Blasko JC, Grimm P et al. Interstitial implantation techniques in prostate cancer. J Surg Oncol 1997; 66: 65-75.

4. Stock RG, Stone NN, Tabert A et al. A dose-response study for I-125 prostate implants. Int J Radiat Oncol Biol Phys 1998; 41: 101-108.

5. Davis BJ, Horwitz EM, Lee WR et al. American Brachytherapy Society consensus guidelines for transrectal ultrasound guided permanent prostate brachytherapy. Brachytherapy 2012; 11: 6-19.

6. Tejwani A, Bieniek E, Lindsay P et al. Case series analysis of post-brachytherapy prostate edema and its relevance to post-implant dosimetry. Post-implant prostate edema and dosimetry. J Contemp Brachytherapy 2012; 4: 75-80.

7. Butzbach D, Waterman FM, Dicker AP. Can extraprostatic extension be treated by prostate brachytherapy? An analysis based on postimplant dosimetry. Int J Radiat Oncol Biol Phys 2001; 51: 1196-1199.

8. Ash D, Al-Qaisieh B, Bottomley D et al. The correlation between D90 and outcome for I-125 seed implant monotherapy for localized prostate cancer. Radiother Oncol 2006; 79: 189-195.

9. Sidhu S, Morris J, Spadinger I et al. Prostate brachytherapy postimplant dosimetry: A comparison of prostate quadrants. Int J Radiat Oncol Biol Phys 2002; 52: 544-552.

10. Maletz KL, Ennis RD, Ostenson J et al. Comparison of CT and MR-CT fusion for prostate post-implant dosimetry. Int J Radiat Oncol Biol Phys 2012; 82: 1912-1917.

11. Yoosuf MAB, Workman G, O'Toole et al. Sector analysis of 125I permanent prostate brachytherapy provides a rapid and effective method of evaluating and comparing pre- and post-implant dosimetry. Brachytherapy 2013; 12: 254-259.

12. Merrick GS, Butler WM, Grimm $P$ et al. Multisector prostate dosimetric quality: Analysis of a large community database. Brachytherapy 2014; 13: 146-151.

13. Sejpal SV, Sathiaseelan V, Helenowski IB et al. Intra-operative pubic arch interference during prostate seed brachytherapy in patients with CT-based pubic arch interference of $<$ or $=1 \mathrm{~cm}$. Radiother Oncol 2009; 91: 249-254.

14. D'Amico AV, Davis A, Vargas SO et al. Defining the implant treatment volume for patients with low risk prostate cancer: does the anterior base need to be treated? Int J Radiat Oncol Biol Phys 1999; 43: 587-590.

15. Merrick GS, Butler WM, Wallner KE et al. Variability of prostate brachytherapy preimplant dosimetry: a multi-institutional analysis. Brachytherapy 2005; 4: 241-251.

16. Lee WR, Roach M3rd, Michalski J et al. Interobserver variability leads to significant differences in quantifiers of prostate implant adequacy. Int J Radiat Oncol Biol Phys 2002; 54: 457-461.

17. Zelefsky MJ, Chou JF, Pei X et al. Predicting biochemical tumor control after prostate brachytherapy for clinically local- 
ized prostate cancer: The Memorial Sloan-Kettering Cancer Center experience. Brachytherapy 2012; 11: 245-249.

18. Morris WJ, Halperin R, Spadinger I. The relationship between post-implant dose metrics and biochemical no evidence of disease following low dose rate prostate brachytherapy: is there an elephant in the room? Brachytherapy 2010; 9: 289-292.

19. D'Souza WD, Thames HD, Kuban DA. Dose-volume conundrum for response of prostate cancer to brachytherapy: summary dosimetric measures and their relationship to tumor control probability. Int J Radiat Oncol Biol Phys 2004; 58: 15401548.

20. Nath S, Chen Z, Yue N et al. Dosimetric effects of needle divergence in prostate seed implant using 125I and 103Pd radioactive seeds. Med Phys 2000; 27: 1058-1066.

21. Partin AW, Mangold LA, Lamm DM et al. Contemporary update of prostate cancer staging nomograms (Partin tables) for the new millennium. Urology 2001; 58: 843-848.

22. Jewett HJ. The present status of radical prostatectomy for stages A and B prostate cancer. Urol Clin North Am 1975; 2: 105-124.

23. Fine SW, Epstein JI. A contemporary study correlating prostate needle biopsy and radical prostatectomy Gleason score. J Urol 2008; 179: 1335-1339.

24. Al-Ahmadie HA, Tickoo SK, Olgac S et al. Anterior-predominant prostatic tumors: Zone of origin and pathologic outcomes at radical prostatectomy. Am J Surg Pathol 2008; 32: 229-235.

25. Merrick GS, Gutman S, Andreini H et al. Prostate cancer distribution in patients diagnosed by transperineal template-guided saturation biopsy. Eur Urol 2007; 52: 715-724.

26. Delongchamps NB, Rouanne M, Flam T et al. Multiparametric magnetic resonance imaging for the detection and localization of prostate cancer: combination of T2-weighted, dynamic contrast enhanced and diffusion-weighted imaging. BJU Int 2011; 107: 1411-1418. 